\title{
Erosion patterns on dissolving and melting bodies
}

\author{
Caroline Cohen, ${ }^{*}$ Michael Berhanu, Julien Derr, and Sylvain Courrech du Pont ${ }^{\dagger}$ \\ Laboratoire Matière et Systèmes Complexes, UMR No. 7057 du CNRS, Université Paris Diderot, \\ 75205 Cedex 13, France
}

(Received 11 August 2016; published 12 September 2016)

\begin{abstract}
This paper is associated with a poster winner of a 2015 APS/DFD Gallery of Fluid Motion Award. The original poster is available from the Gallery of Fluid Motion, http://dx.doi.org/10.1103/APS.DFD.2015.GFM.P0051
\end{abstract}

DOI: 10.1103/PhysRevFluids.1.050508

The dissolution rate of a solid depends on the concentration of the solution at the interface. It consequently depends on the flow, which takes the dissolved material away. The global shape of dissolving bodies has been studied in water at rest [1] and in a high-speed flow [2]. Here we focus on the pattern formation observed on the wall of a dissolving caramel block and on its geomorphogenetic analogs in nature (Fig. 1).

Caramel is an amorphous material with physicochemical properties similar to the glucose used in [1]. Experiments simply consist in putting blocks of solid caramel tilted from the horizontal in a bath of water at rest [Fig. 2(a)]. The block dissolves (the interface recedes at a velocity around $1 \mathrm{~cm} / \mathrm{h}$ ) and patterns emerge underneath the block [Figs. 2(c)-2(f)], whereas the top surface remains smooth. First, longitudinal stripes, with a wavelength of $0.5 \mathrm{~mm}$, appear at short times $(t=10 \mathrm{~s})$. Then the stripes cross and form chevrons $(t=10 \mathrm{~min})$. Chevrons open and evolve toward scallops pointing out downward, which are reminiscent of limestone cave holes modeled by phreatic rivers [3]. After $60 \mathrm{~min}$, the caramel scallops are typically $5 \mathrm{~mm}$ large and $1 \mathrm{~mm}$ deep and travel upward at a velocity of $1 \mathrm{~cm} / \mathrm{h}$.

To understand why the patterns appear, one must consider the release of dissolved caramel. Because it is denser than water, the dissolution induces a buoyancy-driven flow under the block and the heavy layer of fluid destabilizes into sinking filaments. This phenomenon can be seen as a solutal Rayleigh-Bénard instability, where concentration substitutes for temperature, or, identically, as the Rayleigh-Taylor instability of a thin viscous film, whose thickness is controlled by diffusion $[4,5]$. This instability of the flow naturally reflects on the field of concentration at the solid interface. The concentration is higher in the filaments than in between, so the dissolution rate of the solid is lower above filaments. This differential dissolution sculpts the surface. The stripes emerge with the same wavelength as the flow instability, which prints onto the dissolving block. Once formed, the stripes interact with the flow: The filaments are channelized by the crests. In return, the concentration field makes the longitudinal stripes unstable and they evolve toward scallops. On the top surface of the block, the buoyancy is stabilizing. The dissolved caramel flows down along the block, which remains smooth.

Similar patterns can be observed on immersed walls of icebergs in Greenland [Fig. 2(g)]. Ice scallops are typically $20 \mathrm{~cm}$ large and $4 \mathrm{~cm}$ deep and point out upward [Fig. 2(h)]. As the thermodynamic formalism is the same, most dissolution patterns have a melting equivalent [6]. We

*caroline.cohen@univ-paris-diderot.fr

${ }^{\dagger}$ sylvain.courrech@univ-paris-diderot.fr

Published by the American Physical Society under the terms of the Creative Commons Attribution 3.0 License. Further distribution of this work must maintain attribution to the author(s) and the published article's title, journal citation, and DOI. 


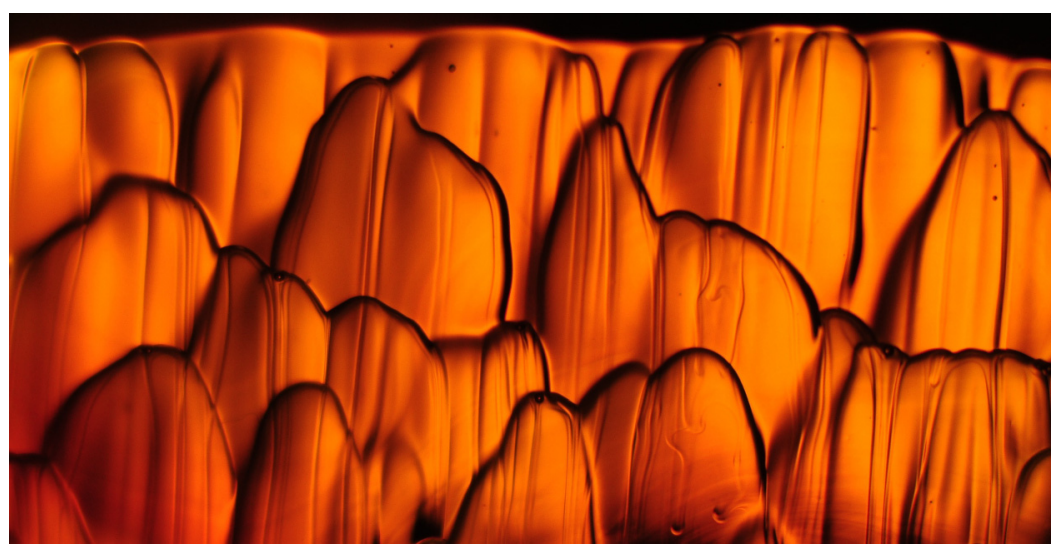

FIG. 1. Patterns observed underneath an inclined block of caramel dissolving in water. Black lines depict the sharp slopes of the holes and thin filaments witness the downward flow of dissolved caramel. DOI: http://dx.doi.org/10.1103/APS.DFD.2015.GFM.P0051

anticipate that the mechanism we described explains scallops on icebergs. Indeed, the freshwater released by the ice melting is less dense than the surrounding sea saltwater. Again, the upward buoyancy-driven flow destabilizes, leading to a heterogeneous salt concentration or temperature, which shapes the iceberg's surface.

Without external flow, the solutal Rayleigh-Bénard instability determines the field of concentration at the wall of a dissolving body, where the buoyancy is destabilizing. There, it leads to the formation of patterns but also controls the overall dissolution rate. The characterization of this mechanism is then of prime geological importance to better understand the erosion processes at work in nature.

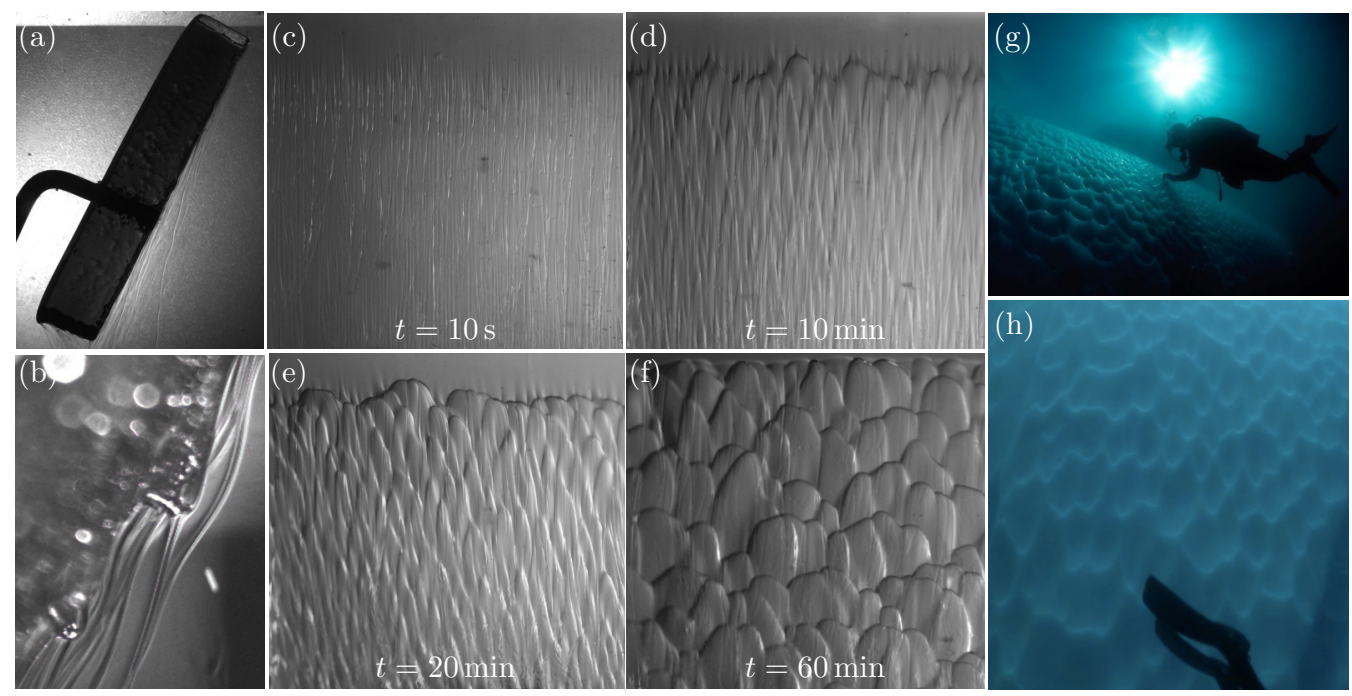

FIG. 2. (a) Side view of the dissolving caramel block. The flowing filaments of dissolved caramel are made visible by the schlieren effect. (b) Side view of the interaction of the scallop pattern and the filaments of caramel. (c)-(f) Patterns observed underneath the block at different times of the dissolution: longitudinal stripes, chevrons, and scallops pointing downward. (g) Scalloping iceberg underwater. (h) Ice scallops pointing upward. (Photographs are from A. Michon.) 


\section{EROSION PATTERNS ON DISSOLVING AND MELTING BODIES}

[1] E. Nakouzi, R. E. Goldstein, and O. Steinbock, Do dissolving objects converge to a universal shape? Langmuir 31, 4145 (2014).

[2] J. M. Huang, M. N. J. Moore, and L. Ristroph, Shape dynamics and scaling laws for a body dissolving in fluid flow, J. Fluid Mech. 765, R3 (2015).

[3] R. L. Curl, Deducing flow velocity in cave conduits from scallops, Natl. Speleogr. Soc. Bull. 36, 1 (1974).

[4] H. R. Brown, Rayleigh-Taylor instability in a finite thickness layer of a viscous fluid, Phys. Fluids A 1, 895 (1989).

[5] L. Limat, Instability of a liquid hanging below a solid ceiling: Influence of layer thickness, C. R. Acad. Sci. Paris Ser. II 317, 563 (1993).

[6] P. Meakin and B. Jamtveit, Geological pattern formation by growth and dissolution in aqueous systems, Proc. R. Soc. London A 466, 659 (2010). 